\title{
DESAFIOS E INOVAÇÃO NA SECAGEM POR MICRO-ONDAS DE MADEIRAS AMAZÔNICAS
}

\section{CHALLENGES AND INNOVATION IN MICROWAVE DRYING OF AMAZONIAN WOOD}

\author{
Anízio de Araújo Cavalcante ${ }^{1}$; Ricardo Manfredi Naveiro ${ }^{2}$ \\ ${ }^{1}$ Universidade do Estado do Amazonas - CESI/UEA \\ aacavalcante@uea.edu.br \\ ${ }^{2}$ Universidade Federal do Rio de Janeiro,- COPPE/UFRJ \\ ricardo.naveiro@poli.ufrj.br
}

\begin{abstract}
Resumo
A secagem desempenha o mais importante papel na industrializaçao da madeira, pois dela depend a qualidade do produto final. $O$ objetivo do presente foi estudar o desempenho de Angelim Vermelho, Muiracatiara e Tauari - Vermelho quanto a secagem por micro-ondas. Os dados foram analisados com transformação $(\sqrt{x})$ e as comparações de médias pelo teste de Tukey, ambas a $5 \%$ de probabilidade. O Angelim - Vermelho com umidade inicial média de 19,78\% alcançou 13,95\% de umidade final média. A Muiracatiara iniciou com 16,88\% de umidade inicial média e obteve $14,7 \%$ de umidade final média. O Tauari - Vermelho com 21,19\% apresentou 15,63\% de umidade final média. $O$ controle de qualidade realizado mostrou a presença de rachaduras de topo e de superfície. Verificou-se através do teste de comparação de médias (Tukey) que as espécies Angelim - Vermelho e Muiracatiara apresentaram comportamento semelhante em relação a racha de superfície. Porém, em relação a rachadura de topo, as madeiras de Muiracatiara e Tauari Vermelho tiveram o mesmo comportamento. A pesquisa mostrou um certo grau de restrições quanto ao conhecimento insuficiente da secagem de madeiras amazônicas pelo processo de microondas.
\end{abstract}

Palavras-chave: secagem de madeiras; micro-ondas; espécies amazônicas.

\section{Introdução}

Um novo processo de secagem de madeiras, utilizando micro-ondas, está em teste no Instituto Nacional de Pesquisas da Amazônia - INPA/INCT - Madeiras da Amazônia. Este método é recente no país, porém seu conceito não é novo, pois foi utilizado pelos nazistas, para cozimento de alimentos, durante a II Grande Guerra, em suas operações de invasão da Rússia (MERCOLA, 2011). A tecnologia de micro-ondas para fins industriais começou a ser utilizada para cozinhar e secar nos idos de 1940, com o advento do magnéton, utilizando frequências de $2450 \mathrm{MHz}$ e 915 MHz (RESCH, 2006). De acordo com Lundgren (2005), o número de aplicações comerciais para a 
utilização de micro-ondas tem aumentado para uma variada gama de aplicações, como aquecimento, comunicações, medições, etc.

A primeira produção empregando secador de ar quente contínuo de micro-ondas, utilizando $50 \mathrm{KW}$ de potência com uma frequência de $915 \mathrm{MHz}$, foi desenvolvida para madeira macia que, mesmo após uma secagem primária, ainda apresentava-se muita úmida (RESCH, 2006). Porém, Egner e Jagfeld (1964) mostram que a tecnologia de secagem por micro-ondas pode ser aplicada aos produtos florestais, principalmente a tábua fina (MCALISTER; RESCH 1971). A seletividade da secagem foi obtida devido à maior absorção de micro-ondas em áreas mais úmidas, muitas vezes definida como bolsa de umidade. Além disso, Resch et al (1970) esclarecem que a resecagem por micro-ondas previne o excesso da secagem e mantém a aparência de qualidade igual ou melhor do que a resecagem com ar quente.

Este processo foi investigado na década de 60, através de pesquisa publicadas de Egner e Jagfeld (1964) e Resch (1968). E mais recente, Antti (1999) procura obter melhor compreensão teórica da secagem por micro-ondas. Contudo, o aquecimento das estufas por micro-ondas apresentam limites para produtos de madeira, pois podem não penetrar profundamente, principalmente quando a madeira está muito úmida, ou não ter um aquecimento uniforme (LUNDGREN, 2005).

No entanto, quando o resultado da distribuição de temperatura não é uniforme, significa dizer que o tamanho do material a ser aquecido é maior que a profundidade de penetração da radiação (RESCH, 2006). Entretanto, o tratamento térmico utilizando micro-ondas oferece oportunidade para o processamento de secagem da madeira em um curto espaço de tempo (ANTTI, 1999), pois é rápido e com possibilidade de ajustes de potência (SEYFART et al 2003). Contudo, investigação na década de 80/90 encontrou problemas, pois este método ainda não está desenvolvido industrialmente em função de uma série de dificuldades técnicas (LEIKER; ADAMSKA, 2004), como por exemplo, problemas de distribuição não homogênea de micro-ondas, além da falta de ferramentas capazes de realizar a medição no interior da estufa (SEYFART et al 2003). Por isso, é necessária e importante a utilização desse processo, pois existe ainda a necessidade da realização de mais pesquisas sobre a aplicação de micro-ondas na secagem da madeira.

Uma qualidade da secagem por micro-ondas é o aquecimento da madeira de dentro para fora, originando calor diretamente no âmago da madeira. Contudo, Kleina (2011) mostra que embora o funcionamento é o de agitar as moléculas de água no interior de um material higroscópico, ocorre justamente o contrário, ou seja o aquecimento começa agitando as moléculas mais externas que movimentam as imediatamente após e, por conseguinte transferindo o calor para o interior do material. 
Por isso, as estufas de micro-ondas podem não ser o ideal para prever a intensidade do aquecimento, pois podem não ser capazes de penetrar profundamente ou ter um aquecimento uniforme. Na região de micro-ondas, a profundidade de penetração se torna muito pequena, principalmente quando a madeira está muito úmida. Quando o resultado da distribuição de temperatura não é uniforme, significa dizer que o tamanho do material a ser aquecido é maior que a profundidade de penetração da radiação (RESCH, 2006).

O tratamento térmico empregando micro-ondas é próspero, pois oferece oportunidade para o processamento de secagem da madeira em um curto espaço de tempo. A energia de micro-ondas pode ser aproveitada com sucesso para o aquecimento rápido e secagem de madeira (ANTTI, 1999), inclusive aumentando sua permeabilidade (TORGOVNIKOV e VINDEN, 2009) e de acordo com Seyfarth et al (2003), a aplicação de micro-ondas reduz o tempo de secagem, pois é rápido e com probabilidade de acertos de potência. Contudo, esta tecnologia ainda não está desenvolvido industrialmente, em função de uma série de dificuldades técnicas.

Ressalta-se que esta tecnologia, em termos de Brasil, é encarada como um processo de inovação, pois ao ser efetivada de maneira ininterrupta traz benefícios a uma empresa a obter benefícios competitivos perante a concorrência. Por isso, o desenvolvimento e a difusão da nova tecnologia de secagem por micro-ondas, desempenha um papel importante para o aumento produtivo de madeira seca. Além da obtenção de madeira seca com qualidade, o processo de secagem por micro-ondas é considerado "amigo do ambiente", pois é uma tecnologia limpa e entendida como todas as tecnologias que são utilizadas na produção de bens e serviços e que não afetam o meio ambiente.

Apesar de ser considerado inédito e inovador para o Brasil, o método de secagem por microondas de madeira amazônicas possui desvantagem e a mais acentuada está na exigência de maior exatidão no cumprimento dos planos de conservação e manutenção observados para o funcionamento dos fornos de micro-ondas. Deve-se ressaltar criteriosamente a condução do processo de secagem, em função principalmente das elevadas temperaturas utilizadas, além da possível radiação das micro-ondas forçando assim, o investimento mais intenso na manutenção dos fornos e também no treinamento de mão de obra. Porém, é de conhecimento que em condições normais, os fornos de micro-ondas possuem níveis de segurança bastante altos. Além disso, sabe-se que a madeira não retém as micro-ondas emitidas pelo forno, pois a única função é o aquecimento das moléculas de água para posterior secagem, e o que fica após o desligamento do forno é o calor. Por isso, a segurança dos aparelhos novos ou em bom estado de conservação e em uso, é a garantia de uma secagem conduzida dentro dos padrões e sem danos ao corpo humano.

No que se refere a estudos sobre o desenvolvimento de secagem por micro-ondas de madeiras no Brasil, a revisão da literatura permite concluir que, devido a carência de estudos, há 
pouquíssimos artigos publicados sobre o tema, além de como esta tecnologia de secagem está sendo executada, indicando que mais pesquisas devem ser exploradas no sentido de melhor conhecimento e divulgação. Além disso, existe ainda a necessidade da realização de mais pesquisas sobre a aplicação de micro-ondas na secagem da madeira, principalmente para as madeiras Amazônicas. Por isso, este trabalho propõe estudar o comportamento da secagem por micro-ondas em relação aos defeitos de rachaduras e colapso das madeiras de Angelim - Vermelho, Muiracatiara e Tauari Vermelho.

\section{Metodologia}

O forno de micro-ondas utilizado é um secador de madeiras (Easydry, Wu. M Gobler, Lichtenau, Alemaha) para $0,7 \mathrm{~m}^{3}$ de madeira empilhada, fabricado com tecnologia alemão (Figura 1). Este secador é projetado para indústrias que utilizam a secagem e o acabamento de madeiras, tais como, fabricantes de móveis, de brinquedos, entalhadores, carpinteiros, restauradores, entre outros.

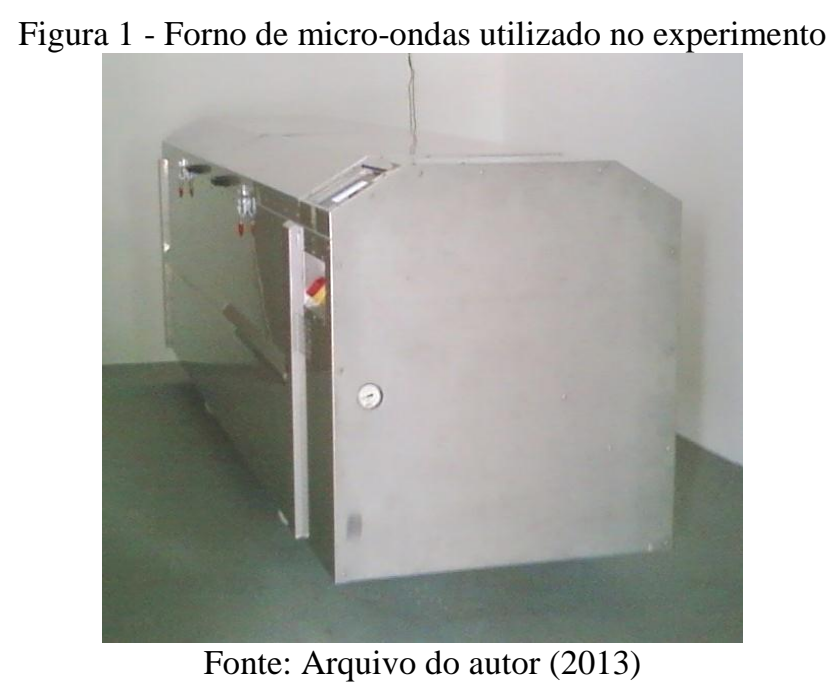

Para realização do presente trabalho, foram utilizadas madeiras de Angelim - Vermelho (Dinizia excelsa), Muiracatiara (Astronium lecontei) e Tauari - Vermelho (Cariniana micrantha) doadas pela Serraria Portela, localizada no Distrito Industrial de Manaus (Am). As tábuas tinham as dimensões nominais de 2,5 cm de espessura, $25 \mathrm{~cm}$ de largura e $95 \mathrm{~cm}$ de comprimento. O Angelim - Vermelho apresenta densidade básica de $0,76 \mathrm{~g} / \mathrm{cm}^{3}$ e índice T/R de 1,67 (REMADE, 2011). A Muiracatiara tem densidade básica de $0,79 \mathrm{~g} / \mathrm{cm}^{3}$ e índice $\mathrm{T} / \mathrm{R}$ de 1,76 , enquanto a espécie de Tauari - Vermelho mostra densidade básica de $0,75 \mathrm{~g} / \mathrm{cm}^{3}$ e um índice T/R de 1,58 (IBAMA, 2011). O software da estufa já vem com 18 programas de secagem de madeiras, e para esta secagem utilizou-se o programa da madeira Nut (Castanha), por apresentar uma densidade entre 0,75 a $0,85 \mathrm{~g} / \mathrm{cm}^{3}$. O Programa de Secagem utilizado apresenta $45{ }^{\circ} \mathrm{C}$ de temperatura, $85 \%$ de 
Umidade Relativa Inicial e 45\% de Umidade Relativa Final. Em função da umidade inicial e final e do peso da carga, o software calcula o tempo de aquecimento, tempo de secagem e tempo de resfriamento e que para esta secagem foram $10 \mathrm{~min}, 4$ horas e 1 hora, respectivamente.

Devido a não existência de programas de secagem por micro-ondas para as espécies amazônicas como Angelim - Vermelho, Muiracatiara e Tauari - Vermelho a pesquisa optou por iniciar a secagem com a temperatura de $45^{\circ} \mathrm{C}$. Porém, Jankowsky et al (1990) esclarecem que a temperatura inicial para secar o Angelim - Vermelho e a Muiracatiara é $40^{\circ} \mathrm{C}$, no entanto para o Tauari - Vermelho a temperatura inicial é $50{ }^{\circ} \mathrm{C}$. Vale ressaltar que essas indicações são referentes a secagem convencional ou a secagem com a utilização de vapor.

Em função do mecanismo de aquecimento de um forno de micro-ondas estar relacionado com a polaridade das moléculas de água que contém nos materiais higroscópicos, e considerando que o aquecimento por micro-ondas ocorre de dentro para fora, foi realizado um teste de distribuição (Figura 2) da umidade na tábua, com a finalidade de observar se a madeira acompanha tal aquecimento e consequentemente tal secagem. A energia que é conduzida pelas micro-ondas para as moléculas de água, originam uma agitação desordenada entre estas moléculas, acrescendo o número de choques entre elas. Este movimento desordenado proporciona o aquecimento e secagem das madeiras.

Figura 2 - Teste de distribuição da umidade em função do aquecimento por micro-ondas
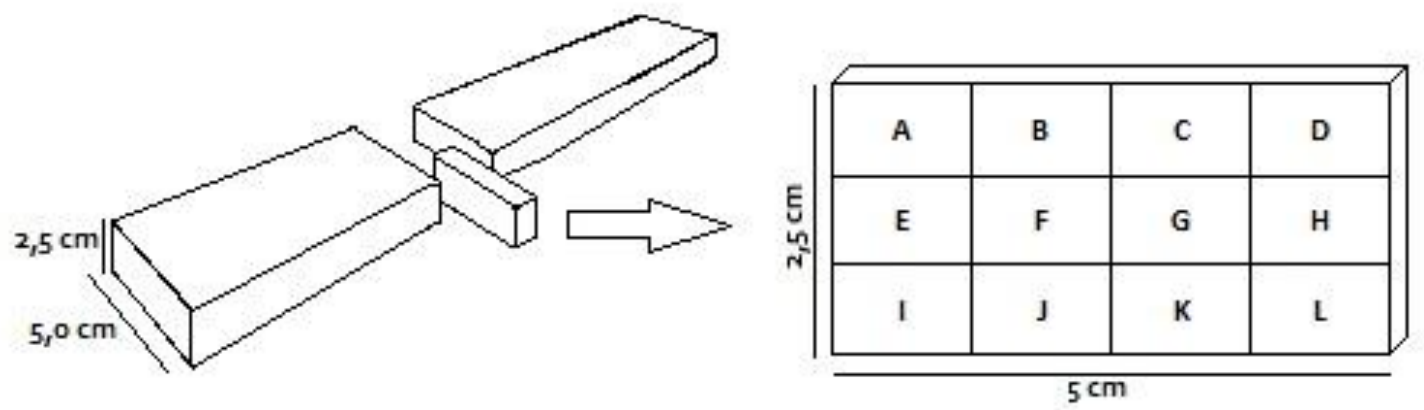

Fonte: Arquivo do autor (2013)

Após a secagem por micro-ondas, os defeitos foram classificados com base na metodologia sugerida por Brasil (1981) e foram avaliados e quantificados de acordo com os critérios estabelecidos na Tabela 1.

Tabela 1 - Critérios utilizados na classificação de tendência do aparecimento de defeitos (colapso, rachas e empenos)

\begin{tabular}{ccc}
\hline Presença do defeito $(\%)$ & Classe & Tendência \\
\hline $0-15$ & 1 & Ausente \\
$16-31$ & 2 & Pequena \\
$32-47$ & 3 & Moderadamente pequena \\
$48-63$ & 4 & Média \\
$64-79$ & 5 & Moderadamente grande \\
$80-100$ & 6 & Grande \\
\hline
\end{tabular}

Fonte: Proposição do autor (2013) 
O estudo foi conduzido em delineamento inteiramente casualizado, com arranjo fatorial, tendo como fatores a duração do tempo de secagem de 4 horas e temperatura fixa de $45{ }^{\circ} \mathrm{C}$. Os dados foram analisados com transformação $(\sqrt{x})$, por análise da variância (ANOVA), pelo teste F e as comparações de médias pelo teste de Tukey, ambas a 5\% de probabilidade.

\section{Resultados e discussão}

O esquema de tratamento de secagem, assim como o teor de umidade inicial (Ui), o teor de umidade final (Uf) e o tempo de secagem (ts) podem ser vistos na Tabela 2.

Tabela 2 - Parâmetros do resultado da secagem por micro-ondas das madeiras de Angelim - Vermelho, Muiracatiara e

\begin{tabular}{c|c|c|c|c}
\hline \multicolumn{5}{c}{ Tauari - Vermelho } \\
\hline Madeira & $\begin{array}{c}\text { Frequência } \\
(\mathrm{GHz})\end{array}$ & $\begin{array}{c}\text { Umidade final Média } \\
(\%)\end{array}$ & Tempo de secagem (hs) \\
\hline Angelim-Vermelho inicial Média (\%) & & 19,78 & 13,95 & $4 \mathrm{~h}$ \\
Muiracatiara & 2,45 & 16,88 & 14,70 & \\
Tauari-Vermelho & & 21,20 & 15,63 & 14,76 \\
Média & & 19,29 & 140
\end{tabular}

Fonte: Pesquisa de campo (2013)

A umidade final programada de $10 \%$ não foi alcançada, contudo a umidade final real foi de aproximadamente $14 \%$. Provavelmente, a umidade inicial tenha sido superestimada, em função de a medição ter sido realizada com a utilização de aparelho elétrico medidor de umidade, que emprega tecnologia eletrônica, com sensores não agressivos. Este processo de verificação da umidade permite a medição pelo simples toque dos sensores na superfície da madeira, no entanto o valor obtido diz respeito a uma profundidade de até $50 \mathrm{~mm}$.

Outra observação foi que as tábuas utilizadas no estudo não mostraram mudança de cor, que é uma consequência provocada pela ausência de oxigênio durante o tempo de secagem. Nota-se que os resultados encontrados são similares aos notados por Seyfarth et al (2003) por ocasião da secagem da madeira de faia e de carvalho. 
Tabela 3 - Amostra de distribuição de umidade após secagem

\begin{tabular}{|c|c|c|c|}
\hline 14,54 & 14,56 & 14,58 & 14,36 \\
\hline 13,76 & 12,62 & 12,76 & 13,56 \\
\hline 14,30 & 14,82 & 14,70 & 14,54 \\
\hline
\end{tabular}

\begin{tabular}{|c|c|c|c|}
\hline 13,62 & 13,50 & 13,38 & 13,42 \\
\hline 13,56 & 11,78 & 12,10 & 13,62 \\
\hline 13,66 & 13,92 & 13,64 & 13,66 \\
\hline
\end{tabular}

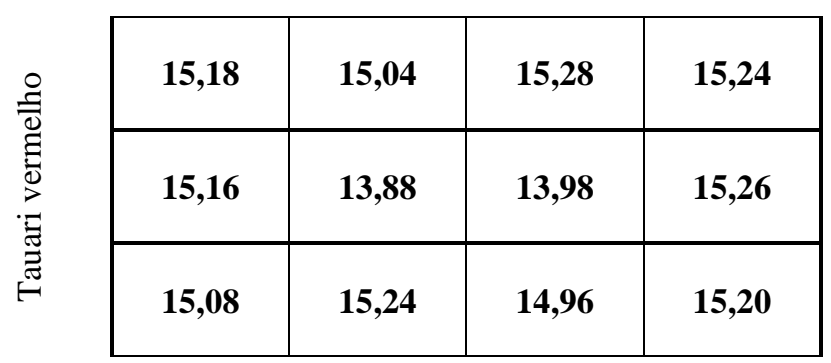

Fonte: Pesquisa de campo (2013)

Observa-se na Tabela 3 que a distribuição da umidade tanto na espessura quanto na largura de uma peça de madeira, está de acordo com o aquecimento e secagem por micro-ondas. Nota-se que as maiores umidades encontram-se na parte externa das amostras de madeira.

Como o principal objetivo deste trabalho foi à verificação de defeitos do tipo colapso, rachaduras de superfície, de topo e interna por meio da secagem por micro-ondas, será apresentada a avaliação e análise dos resultados obtidos. Assim como a diferença de retratibilidade para esta espécie está um pouco acima da T/R de 1,5, é esperado que os problemas decorrentes da secagem ocorressem.

O controle de qualidade efetuado antes da secagem apresentou tábuas isentas de defeitos, e, após secagem, a análise mostrou que não houve a presença de colapso e nem rachadura interna, indicando que provavelmente a ausência desses defeitos esteja relacionada com a utilização do programa empregado, levando a crer que o programa não provocou a formação de qualquer tipo de colapso: nem suave e nem severo.

A Tabela 4 apresenta os valores de umidade (inicial e final), o percentual de perda de água e os valores dos escores dos defeitos analisados, onde se observou que nas secagens efetuadas, houve somente a presença de rachas de superfície e de topo. É possível observar que o Angelim vermelho apresentou a maior quantidade de peças, tanto com rachadura de topo quanto rachadura de superfície, apesar de ter 19,78\% de umidade inicial. Por um lado, a Muiracatiara, não obstante 
ter apresentado umidade inicial média de 16,88\%, mostrou-se mais propenso a rachadura de superfície do que a rachadura de topo. Por outro lado, o Tauari - vermelho que iniciou com a maior umidade inicial média de $21,20 \%$, é a espécie que pode ser considerada isenta na formação das rachaduras, pois mostrou apenas rachadura de topo, e em pequena quantidade.

Tabela 4 - Valores da umidade inicial e final e escores dos defeitos da madeira de Angelim - vermelho, Muiracatiara e Tauari - vermelho

\begin{tabular}{|c|c|c|c|c|c|}
\hline Amostra & $\mathrm{Ui}$ & UF & $\%$ perda de água & RT & $\mathrm{RS}$ \\
\hline Angelim Vermelho & 21.1 & 18.3 & 13.3 & 3 & 3 \\
\hline Angelim Vermelho & 23.8 & 16.9 & 29.0 & 3 & 1 \\
\hline Angelim Vermelho & 22.2 & 15.7 & 29.3 & 1 & 3 \\
\hline Angelim Vermelho & 14.7 & 9.4 & 36.1 & 3 & 3 \\
\hline Angelim Vermelho & 21.5 & 14.5 & 32.6 & 3 & 3 \\
\hline Angelim Vermelho & 20.5 & 13.4 & 34.6 & 1 & 3 \\
\hline Angelim Vermelho & 23 & 15.6 & 32.2 & 3 & 1 \\
\hline Angelim Vermelho & 16.3 & 10.8 & 33.7 & 1 & 3 \\
\hline Angelim Vermelho & 20.6 & 14.8 & 28.2 & 1 & 1 \\
\hline Angelim Vermelho & 14.4 & 10.1 & 29.9 & 3 & 1 \\
\hline Angelim Vermelho & 21.9 & 14.4 & 34.2 & 1 & 3 \\
\hline Angelim Vermelho & 21.6 & 14.4 & 33.3 & 3 & 3 \\
\hline Angelim Vermelho & 14.7 & 10.0 & 32.0 & 1 & 1 \\
\hline Angelim Vermelho & 13.6 & 9.5 & 30.1 & 3 & 1 \\
\hline Angelim Vermelho & 19.9 & 16.0 & 19.6 & 1 & 3 \\
\hline Angelim Vermelho & 18.6 & 14.1 & 24.2 & 3 & 3 \\
\hline Angelim Vermelho & 21.5 & 14.5 & 32.6 & 1 & 1 \\
\hline Angelim Vermelho & 21.6 & 16.0 & 25.9 & 3 & 1 \\
\hline Angelim Vermelho & 19.6 & 13.5 & 31.1 & 1 & 3 \\
\hline Angelim Vermelho & 24.4 & 17.1 & 29.9 & 3 & 3 \\
\hline Muiracatiara & 14.2 & 12.4 & 12.7 & 2 & 2 \\
\hline Muiracatiara & 15.8 & 14.6 & 7.6 & 2 & 1 \\
\hline Muiracatiara & 15.1 & 14.3 & 5.3 & 1 & 3 \\
\hline Muiracatiara & 18.3 & 15.5 & 15.3 & 2 & 2 \\
\hline Muiracatiara & 17.6 & 15.1 & 14.2 & 1 & 1 \\
\hline Muiracatiara & 15.6 & 14.2 & 9.0 & 1 & 3 \\
\hline Muiracatiara & 17.6 & 13.6 & 22.7 & 1 & 1 \\
\hline Muiracatiara & 17.9 & 12.2 & 31.8 & 1 & 3 \\
\hline Muiracatiara & 16.9 & 15.4 & 8.9 & 3 & 3 \\
\hline Muiracatiara & 18.7 & 14.1 & 24.6 & 1 & 1 \\
\hline Muiracatiara & 17.7 & 15.6 & 11.9 & 1 & 3 \\
\hline Muiracatiara & 18.1 & 17.0 & 6.1 & 2 & 2 \\
\hline Muiracatiara & 13.6 & 11.7 & 14.0 & 1 & 3 \\
\hline Muiracatiara & 17.1 & 16.4 & 4.1 & 1 & 3 \\
\hline Muiracatiara & 16.7 & 14.9 & 10.8 & 1 & 1 \\
\hline Muiracatiara & 18.7 & 17.0 & 9.1 & 1 & 1 \\
\hline Muiracatiara & 15.9 & 13.5 & 15.1 & 1 & 1 \\
\hline Muiracatiara & 19.6 & 16.8 & 14.3 & 2 & 2 \\
\hline Muiracatiara & 15.8 & 15.5 & 1.9 & 1 & 3 \\
\hline Muiracatiara & 16.7 & 14.2 & 15.0 & 1 & 3 \\
\hline Tauari vermelho & 21.5 & 17.3 & 19.5 & 1 & 1 \\
\hline Tauari vermelho & 21.3 & 17.6 & 17.4 & 1 & 1 \\
\hline Tauari vermelho & 19.5 & 17.1 & 12.3 & 1 & 1 \\
\hline Tauari vermelho & 21.4 & 16.1 & 24.8 & 1 & 1 \\
\hline Tauari vermelho & 21.3 & 16.3 & 23.5 & 1 & 1 \\
\hline Tauari vermelho & 19.8 & 13.7 & 30.8 & 1 & 1 \\
\hline Tauari vermelho & 21.5 & 14.2 & 34.0 & 1 & 1 \\
\hline Tauari vermelho & 18.9 & 17.3 & 8.5 & 2 & 1 \\
\hline Tauari vermelho & 21.9 & 17.2 & 21.5 & 1 & 1 \\
\hline Tauari vermelho & 21.5 & 15.1 & 29.8 & 1 & 1 \\
\hline Tauari vermelho & 22.1 & 16.3 & 26.2 & 1 & 1 \\
\hline
\end{tabular}




$\begin{array}{lrrrrr}\text { Tauari vermelho } & 22.7 & 16.6 & 26.9 & 1 & 1 \\ \text { Tauari vermelho } & 20.5 & 16.0 & 22.0 & 1 & 1 \\ \text { Tauari vermelho } & 18.8 & 13.8 & 26.6 & 1 & 1 \\ \text { Tauari vermelho } & 20.9 & 13.5 & 35.4 & 1 & 1 \\ \text { Tauari vermelho } & 21.5 & 16.9 & 21.4 & 2 & 1 \\ \text { Tauari vermelho } & 22.2 & 14.0 & 36.9 & 1 & 1 \\ \text { Tauari vermelho } & 23.6 & 12.2 & 48.3 & 1 & 1 \\ \text { Tauari vermelho } & 22.3 & 15.6 & 30.0 & 1 & 1 \\ \text { Tauari vermelho } & 20.7 & 15.7 & 24.2 & 1 \\ \text { Ui = Umidade inicial; Uf = Umidade final; RS = Rachadura de Superfície; RT = Rachadura de } \\ \text { Topo. } \\ \text { Fonte: Pesquisa de campo (2013) }\end{array}$

Os resultados das avaliações dos defeitos da secagem das 3 (três) espécies são mostrados na Tabela 5, onde se verifica a classificação de tendência do aparecimento de defeitos (Tabela 1) em função do número de tábuas provenientes das secagens realizadas. É importante observar que a espécie Angelim - Vermelho $(\mathrm{T} / \mathrm{R}=1,67)$ apesar de apresentar tendência moderada ao torcimento e ao colapso, apresentou média tendência a formação de rachadura de superfície e também de rachadura de topo. Provavelmente, a temperatura utilizada no experimento aliada a taxa T/R para esta espécie tenha sido o principal fator para a formação das rachaduras. A espécie Muiracatiara $(\mathrm{T} / \mathrm{R}=1,76)$ apresenta média tendência a formação de defeitos de rachadura superficial e de pequena tendência a rachadura de topo. Porém, é perfeitamente aceitável este resultado, pois a espécie Muiracatiara exibe tendência moderada as rachaduras fortes. Observa-se que estes defeitos podem ter ocorrido em função da temperatura inicial de secagem, por ter sido maior do que a sugerida por Jankowsky et al (1990). Conforme Aguiar e Jankowsky (1986) a rachadura de superfície e de topo não é uma função das tensões de crescimento, porquanto ocorre em função do movimento superior à resistência da madeira entre a direção radial e a direção tangencial através da secagem e é dependente das espécies, porém de intensidades diferentes.

A madeira durante uma secagem seja convencional ou não, pode sofrer defeitos, como empenamentos e rachaduras. Contudo, Torgovnikov e Vinden (2009) esclarecem que dependendo da espécie de madeira, da espessura e do teor de umidade inicial, o processo com energia por microondas é recomendado, todavia existe a possibilidade do desenvolvimento de rachaduras internas e externas.

Verifica-se que a espécie Tauari - Vermelho, seca com temperatura igual a sugerida por Jankowsky et al (1990), apresentou o mínimo de peças com rachadura de topo e nenhuma com rachadura de superfície, apesar de mostrar disposição a rachadura de superfície, encanoamento, torcimento e encurvamento médios. Provavelmente o baixo valor da T/R do Tauri - Vermelho (T/R $=1,58$ ), aliado a temperatura de secagem, tenham contribuído para a pouca ou a não formação dos defeitos denominados rachaduras.

Ao se analisar as espécies em conjunto verifica-se que o Angelim - Vermelho e a Muiracatiara mostraram tendência a formação de rachaduras, enquanto o Tauari - Vermelho 
mostrou-se totalmente isento aos defeitos.

Tabela 5 - Avaliação dos defeitos de secagem por micro-ondas para o número de peças utilizadas $\left(\mathrm{n}^{\circ}\right)$ das madeiras de $^{\circ}$ Angelim - Vermelho (AV), Muiracatiara (MT) e Tauari - Vermelho (TV)

\begin{tabular}{|c|c|c|c|c|c|c|c|}
\hline \multirow{3}{*}{ Defeitos } & \multirow{2}{*}{$\frac{\text { Experimentos }}{\text { U\% Média }}$} & \multicolumn{2}{|c|}{$\begin{array}{c}\mathrm{AV} \\
\left(45^{\circ} \mathrm{C}\right)\end{array}$} & \multicolumn{2}{|c|}{$\begin{array}{c}\mathrm{MT} \\
\left(45^{\circ} \mathrm{C}\right)\end{array}$} & \multicolumn{2}{|c|}{$\begin{array}{c}\text { TV } \\
\left(45^{\circ} \mathrm{C}\right)\end{array}$} \\
\hline & & \multicolumn{2}{|c|}{19,78} & \multicolumn{2}{|c|}{16,88} & \multicolumn{2}{|c|}{21,20} \\
\hline & Critérios & $\mathrm{n}^{\mathrm{o}}$ & $\%$ & $\mathrm{n}^{\mathrm{o}}$ & $\%$ & $\mathrm{n}^{\mathrm{o}}$ & $\%$ \\
\hline Colapso & 1 & 20 & 100 & 20 & 100 & 20 & 100 \\
\hline$\%$ Total de defeito & & & $\mathbf{0}$ & & $\mathbf{0}$ & & $\mathbf{0}$ \\
\hline Rachadura interna & 1 & 20 & 100 & 20 & 100 & 20 & 100 \\
\hline$\%$ Total de defeito & & & $\mathbf{0}$ & & $\mathbf{0}$ & & $\mathbf{0}$ \\
\hline & 1 & 8 & 40 & 7 & 35 & 20 & 100 \\
\hline Rachadura de superfície & 2 & 0 & 0 & 4 & 20 & 0 & 0 \\
\hline & 3 & 12 & 60 & 9 & 45 & 0 & 0 \\
\hline$\%$ Total de defeito & & & 60 & & 65 & & $\mathbf{0}$ \\
\hline Rachadura de tono & 1 & 9 & 45 & 14 & 70 & 18 & 90 \\
\hline Kacnadura de topo & 2 & 0 & 0 & 5 & 25 & 2 & 10 \\
\hline & 3 & 11 & 55 & 1 & 5 & 0 & 0 \\
\hline$\%$ Total de defeito & & & 55 & & 30 & & 10 \\
\hline
\end{tabular}

Fonte: Pesquisa de campo (2013)

A aferição pelo teste de Tukey consiste em examinar se os resultados determinados são equivalentes aos alcançados experimentalmente. Deve-se verificar se os valores dos defeitos de rachadura de topo e rachadura de superfície ocorrida para as espécies na secagem por micro-ondas são equivalentes para as espécies estudadas experimentalmente.

Nas secagens realizadas, foi encontrada uma amplitude em relação ao tipo de teste empregado (Tabela 2). A elevada aplicação do teste de Tukey pode ser um indicativo da rigorosidade desejada pelos pesquisadores no momento da escolha das espécies que apresentaram menores quantidades de defeitos. Entretanto, não raras vezes, esta rigorosidade reflete dificuldades na interpretação dos testes, devido à classificação dos tratamentos em muitos grupos, o que interfere nas interpretações e conclusões emitidas. Contudo, é importante assegurar através do teste de Tukey qual espécie, ou quais espécies apresentam a menor ou nenhuma tendência aos defeitos de rachadura de topo e rachadura de superfície.

A Tabela 6 apresenta as Comparações de médias das secagens dos dados médios dos defeitos de rachadura de superfície e de topo para as madeiras pesquisadas de Angelim - Vermelho, Muiracatiara e Tauari - Vermelho. Em geral, as secagens do Angelim - Vermelho e da Muiracatiara não mostraram comportamento diferente, em função das médias do defeito apresentado de rachaduras de superfície. No entanto, ao se comparar as médias do defeito rachadura de topo, é importante notar que apesar das secagens mostrarem comportamento semelhante, a secagem das espécies Muiracatiara e Tauari - Vermelho mostraram menor média percentual de rachadura de topo. 
Tabela 6 - Comparação das espécies Angelim - Vermelho (AV), Muiracatiara (MT) e Tauari - Vermelho (TV) para os defeitos Rachadura de Superfície e de Topo

\begin{tabular}{cccccc}
\hline \multicolumn{3}{c}{ Rachadura de Superfície } & \multicolumn{3}{c}{ Rachadura de Topo } \\
\hline Secagem & Média & Tukey & Secagem & Média & Tukey \\
\hline AV & 1,439 & a & AV & 1,403 & a \\
MT & 1,412 & a & MT & 1,140 & b \\
TV & 1,000 & b & TV & 1,041 & b \\
\hline
\end{tabular}

Médias com a mesma letra não diferem entre si.

Fonte: Pesquisa de campo (2013)

É importante ressaltar que a madeira antes da secagem pode apresentar um potencial que se pode aumentar a taxa de secagem, reduzindo os defeitos de secagem e consequentemente aumentando o seu rendimento. Machado (2006) mostra que a secagem por micro-ondas da madeira de carvalho, pode reduzir o estoque total e tempo de processamento para cerca de 12 dias, com reduções concomitantes na manipulação de madeira, estoque e custos operacionais. Apesar de não ter sido abordado neste estudo, mas a redução do tempo de secagem leva a uma redução significativa no consumo de energia, possibilitando a diminuição do tempo de produção.

\section{Considerações finais}

Com base nos resultados da pesquisa da secagem por micro-ondas do Angelim - vermelho, Muiracatiara e Tauari - vermelho, será realizado um resumo do que foi tratado neste trabalho. O presente trabalho inicia com os conceitos da secagem por micro-ondas. O importante da pesquisa será o despertar do conhecimento e o repasse dessa tecnologia a indústria madeireira, apesar da literatura abordar os projetos de secagem de madeiras europeias. Porém, justifica-se que um fator de possível sucesso neste tipo de secagem será o conhecimento e emprego por todos que trabalham a madeira. A pesquisa sobre a secagem por micro-ondas deve ser dividida, na seleção das espécies, na determinação de programas de secagem e o gasto de energia elétrica.

A idealização da análise adotada foi caracterizada como pesquisa prática, uma vez que possui como alvo a determinação de programas da temperatura de secagem. Foi mostrada a temperatura ideal utilizada na secagem convencional, como a descrição da qualidade final da madeira. No entanto, no Brasil esta tecnologia é recente, e a micro-ondas melhora as propriedades físicas, reduzindo o impacto ambiental em seu processamento. Por isso, é importante que as empresas comecem a utilizar esse método de secagem para produzir de forma aceitável os produtos de madeira.

O experimento propriamente dito começou analisando a distribuição da umidade na amostra após secagem, onde foi verificado que o aquecimento por micro-ondas começa a ocorrer no interior da madeira. Verifica-se que o aquecimento e secagem seguiu o comportamento de aquecimento das micro-ondas, pois as umidades foram maiores na parte externa das amostras. Quando da análise dos defeitos, observou-se em geral que as madeiras de Angelim - vermelho, Muiracatiara e Tauari - 
vermelho iniciaram com a umidade média de 19,29\% e finalizaram com a umidade final média de $14,76 \%$, tendo o processo de secagem ocorrido em 4 horas. A análise efetuada no resultado geral das espécies utilizadas mostrou que a madeira de Angelim - Vermelho apresenta média tendência a formação de rachadura de superfície e rachadura de topo. Por outro lado, a madeira de Muiracatiara mostrou tendência moderadamente grande a formação da rachadura de superfície e pequena tendência ao desenvolvimento da rachadura de topo, enquanto a espécie Tauari - Vermelho não apresentou tendência a nenhum defeito. Todas as espécies não apresentaram colapso e nem rachadura interna e também não houve a descoloração das madeiras. Observam-se nesta análise que foi realizada comparações de médias (teste Tukey) nas secagens dos dados sobre os defeitos ocorridos. Verifica-se que as espécies Angelim - vermelho e Muiracatiara não mostraram desempenho distinto em relação a diferença de rachadura. Por outro lado, as espécies de Muiracatiara e Tauari - vermelho mostraram mesmo comportamento quando relacionado ao defeito rachadura de topo.

Analisando que o estudo efetivado possui contribuições reais ao campo das engenharias de produção e florestal, pois os subsídios ocasionados é a proposição do modelo, que se espera ser utilizado para apoiar futuros estudos no aperfeiçoamento e determinação de novos programas de secagem por micro-ondas de espécies amazônicas. O presente estudo apresentou algumas limitações, como por exemplo, o pouco conhecimento da secagem de madeiras pelo processo por micro-ondas, como também a utilização de madeiras amazônicas.

Portanto, recomendam-se pesquisas com secagem por micro-ondas para mais espécies, pois provavelmente há vantagens em se utilizar este método na secagem de madeiras amazônicas. Além disso, está sendo avaliado o uso mais agressivo do tempo de micro-ondas com a finalidade de atingir reduções no tempo de secagem.

\begin{abstract}
The drying plays the most important role in the wood manufacturing, as it depend quality of the final product. The aim of the present was to study the performance of Angelim - Vermelho, Muiracatiara and Tauari - Vermelho woods as microwave drying. Data were analyzed using transform $(\sqrt{x})$ and comparisons of means by Tukey test, both the $5 \%$ probability. The Angelim Vermelho with initial moisture reached $19.78 \%$ and $13.95 \%$ of final moisture. The Muiracatiara started with $16.88 \%$ of initial moisture and obtained $14.7 \%$ of average final moisture. The Tauari Vermelho with $21.19 \%$ had $15.63 \%$ of final moisture. The quality carried out before drying does not detected defects, but after it was noted the presence of top and the surface cracks. It was verified by testing for mean comparison (Tukey) that species Angelim - Vermelho and Muiracatiara showed similar behavior against the crack surface. However, in relation to top crack Muiracatiara and Tauari - Vermelho woods had the same behavior. The research has shown a degree of restrictions to insufficient knowledge of the drying process by microwave for Amazonian woods.
\end{abstract}

Keywords: wood drying; microwave; amazonian species. 


\section{Referências}

AGUIAR, O.J.R.; JANKOWSKY, I.P. Prevenção e controle das rachaduras de topo em tora de Eucalyptus grandis Hill ex Maiden. Piracicaba: IPEF 33:.39 - 46. 1986.

ANTTI, L. Heating and drying wood using microwave power. $\mathrm{PhD}$ Thesis, Luleå University of Technology, Skelleftea, Sweden. 1999.

BRASIL. Superintendência do Desenvolvimento da Amazônia. Estudos sobre métodos de secagem de madeiras da Amazônia. Belém: SUDAM, p. 1-18. 1981.

EGNER, K.; JAGFELD, P. Versuche zur künstlichen Trocknung von Holtz durch Mikrowellen. HoltzZentralblatt, 129: 297-300. 1964.

IBAMA, M. B. 2011. Disponível em:

<http://www.ibama.gov.br/lpf/madeira/caracteristicas.php?ID=86\&característica=25>. Acesso em: 04/04/2011.

JANKOWSKY, I.P.: CHIMELO, J.P.; CAVALCANTE, A.A.; GALINA, I.C.M.; NAGAMURA J.C.S. Madeiras Brasileiras. Ed. Spectrum. Caxias do Sul. 171 p. 1990.

KLEINA, N. 2011. Mitos e Verdades sobre o Micro-ondas. Disponível em: <http://www.tecmundo.com.br/eletrodomesticos/10978-mitos-e-verdades-sobre-o-micro-ondas.htm>. Acesso em: 01.08.2013.

LEIKER, M.; ADAMSKA, M.A. Energy efficiency and drying rates during vacuum microwave drying of wood. Holz Roh Werkst, n. 62, p. 203-208. 2004.

LUNDGREN, N. Modelling microwave measurements in wood. Licentiate Thesis, Luleå University of Technology, Skellefteå, Sweden. 65 p. 2005.

MACHADO, J.S. Effect of microwave treatment on oak compression strength. Silva Lusitanica, v. 14, n. 1, p. 51-58. 2006.

MCALISTER, W.R.; RESCH, H. Drying 1-inch ponderosa pine lumber with a combination of microwave power and hot air. Forest Products Journal, v. 21, n. 3, p. 26-34. 1971.

MERCOLA. 2011. Madeiras. Disponível em: <http://www.mercola.com/article/ microwave/ hazards.htm>. Acesso em 09 ago. 2011.

REMADE. R. M.. 2011. Disponível em: <http://www.remade.com.br/br/madeira_

especies.php?num=114\&title=\&especie=Angelim - Vermelho $>$. Acesso em: 09 de ago. 2011.

RESCH, H. Microwaves for the drying of wood products. Holz als Roh- und Werkstof, v.6, n. 9, p. 317-324. 1968.

RESCH, H. High-frequency electric current for drying of wood - Historical perspectives. Maderas: Ciencia y tecnologia, v. 8, n. 2, p. 67-82. 2006.

RESCH, H.; LOFDAHL, C.A.; SMITH, F.J.; ERB, C. Moisture leveling in veneer by microwaves and hot air. Forest Products Journal, v. 20, n. 10, p. 50-58. 1970.

SEYFARTH, H. R.; LEIKER, M.; MOLLEKOPT, M. Continuous drying of lumber in a vacuum kiln. In: $8^{\text {th }}$ International IUFR, p. 159-163. 2003.

TORGOVNIKOV, G; VINDEN, P. High-intensity microwave wood modification for increasing permeability. Forest Products Journal, v. 59, n. 4, p. 84-92. 2009.

\section{Dados dos autores}

Nome completo: Anízio de Araújo Cavalcante 
Filiação institucional: Universidade do Estado do Amazonas - CESI/UEA

Função ou cargo ocupado: Engenheiro Operacional da Indústria da Madeira, Doutorando em Engenharia de Produção.

Endereço completo para correspondência (bairro, cidade, estado, país e CEP): Centro de Ensino Superior de Itacoatiara da Universidade do Estado do Amazonas - CESI/UEA, Rua Mário Andreazza, s/n, São Francisco, 69.000-000, Itacoatiara, AM.

Telefones para contato: (92) 9614-1807

e-mail: aacavalcante@uea.edu.br

Nome completo: Ricardo Manfredi Naveiro

Filiação institucional: Instituto Alberto Luiz Coimbra de Pós Graduação e Pesquisa de Engenharia da Universidade Federal do Rio de Janeiro

Departamento: Engenheiro Mecânico, Professor, Doutor em Projeto do Produto

Endereço completo para correspondência (bairro, cidade, estado, país e CEP): Instituto Alberto Luiz Coimbra de Pós Graduação e Pesquisa de Engenharia da Universidade Federal do Rio de Janeiro COPPE/UFRJ, Cidade Universitária - Centro de Tecnologia, Cidade Universitária 21.945-970, Rio de Janeiro, RJ,

Telefones para contato: (21) 2562-8253,

e-mail: ricardo.naveiro@poli.ufrj.br.

Submetido em: 01/08/2013

Aceito em: 17/09/2014 